\title{
Enological Behaviour of Biofilms Formed by Genetically-Characterized Strains of Sherry Flor Yeast
}

\author{
María E. Rodríguez ${ }^{1, \dagger}$, Juan J. Infante ${ }^{2, \dagger}$, Juan J. Mesa ${ }^{3, \dagger}$, Laureana Rebordinos ${ }^{1}$, and Jesús M. \\ Cantoral $^{1, *}$ \\ ${ }^{1}$ Laboratorio de Microbiología y Genética, CASEM, Departamento de Biomedicina, Biotecnología y Salud Pública. \\ Universidad de Cádiz. Polígono Río San Pedro s/n, 11510 Puerto Real, Cádiz, Spain \\ ${ }^{2}$ Bioorganic Research and Services, S.L.-Bionaturis, Centro Andaluz de Biología del Desarrollo, Sevilla, Spain \\ ${ }^{3}$ Bodegas Barbadillo S. L. C/ Luis Eguilar, 11540, Sanlúcar de Barrameda, Cádiz, Spain
}

\begin{abstract}
The flor yeasts (Saccharomyces cerevisiae) form a biofilm, known as flor velum, on the surface of fino-type sherry wine at the end of the alcoholic fermentation. These film-forming yeasts are responsible for the oxidative transformation of alcohol to acetaldehyde, together with other reactions, which produce the characteristic flavours and aromas of these wines. In this study, we examine the enological behaviour of eight flor yeast strains biofilms in biological aging experiments carried out in the laboratory. Strains with identical chromosomal and mitochondrial DNA patterns and the same origin showed a more closely-related enological behaviour. But the kinetics of growth and acetaldehyde accumulation in the wine were found to be strain-dependent. Moreover, some strains were marked by high acetaldehyde accumulation in their pure cultures during the various phases of the biofilm development. These results provide valuable knowledge for planning technical strategies to improve the biological aging process in the sherry wine industry.
\end{abstract}

Keywords: flor yeast, sherry wines, pure culture, biofilm growth, acetaldehyde.

\section{INTRODUCTION}

The sherry wine-making process is characterized by the addition of alcohol to the freshly-fermented wine to raise the concentration of ethanol to $15 \%(\mathrm{v} / \mathrm{v})$. The wine, which is depleted in fermentable sugars after the fermentation phase, is then aged in partially filled $600 \mathrm{~L}$ oak barrels. During the aging process a spontaneous biofilm of yeast (flor yeast) grows on the surface of the wine inside the barrels.

The initial studies conducted to differentiate between strains of flor yeasts tested the ability of the yeast clones to assimilate and ferment different sugars. Based on these criteria, the flor yeasts of the Jerez region of South-western Spain were split into four different races of the $S$. cerevisiae species, denominated beticus, cheresiensis, montuliensis, and rouxii [1]. Since then, molecular methods have been used to characterize the flor and other industrial yeasts of the Jerez region [2-6]. These techniques are based on polymorphisms in the number and size of chromosomes and on the restriction patterns of the mitochondrial DNA (mtDNA). However, although these molecular techniques have been available to the wine producers for several years, the biological aging of

\footnotetext{
*Address correspondence to this author at the Laboratorio de Microbiología y Genética, CASEM, Departamento de Biomedicina, Biotecnología y Salud Pública. Universidad de Cádiz. Polígono Río San Pedro s/n, 11510 Puerto Real, Cádiz, Spain; Tel.: +34 9560161 56; Fax: +34 95601 61 80; E-mail: jesusmanuel.cantoral@uca.es

${ }^{\top}$ These authors have contributed equally to this work.
}

Sherry wines is not yet subjected to microbiological control in the wineries.

The aerobic metabolism of flor yeasts influences the characteristics flavour and aroma compounds of the finished sherry [7]. In particular, the acetaldehyde released into the wine by the yeast is considered to be the most important index of aging in sherry wines [8].

The length of the aging period and the large number of oak barrels required in the industrial system represent considerable costs for the producer. One of the more significant costs is that associated with the periodic replenishment of the ethanol consumed by the flor yeasts. This action is critical to prevent the wine from being spoilt by other biofilm-forming yeasts that might grow at ethanol concentrations lower than $15 \%(\mathrm{v} / \mathrm{v})$. The optimum length of the aging period could be shortened by selecting flor yeast strains capable of inducing a more rapid accumulation of the metabolites responsible for the sensory properties of the wine, and then using these strains to inoculate all the wine aging in the winery's barrels. Therefore, the potential association of specific molecular patterns with differences in the performance of the flor yeasts under industrial conditions suggests that molecular methods might be particularly appropriate for identifying and monitoring flor yeast strains with the object of controlling and improving the aging process. This possibility has also been supported by the molecular characterization of different sherry wine aging systems reported by other authors $[9,10]$. 
In this study, we selected eight strains of Saccharomyces cerevisiae flor yeast, which had previously been defined on the basis of their electrophoretic karyotypes and mitochondrial DNA restriction profiles, in order to examine the behaviour of the biofilm in pure cultures during a long period of sherry wine aging. We carried out the assays under laboratory conditions imitating the industrial process. The accumulation of acetaldehyde in the wine, the consumption of ethanol and volatile acidity, growth kinetics and development of the biofilm were the critical aspects of the process analyzed. We then examined the correspondence between enological behaviour and molecular patterns of the strains. Based on the results of this study, we propose a strategy for improving the industrial biological aging fino-type sherry wines by selecting the most suitable flor yeast strain.

\section{MATERIALS AND METHODS}

\section{Yeast Strains}

The $S$. cerevisiae yeast strains used in this study and their origin are shown in Table $\mathbf{1}$. The eight yeasts were isolated from both static and dynamic industrial aging systems in a winery located at the Jerez region in southern Spain. Añada A and Añada B were two static systems for aging the sherry, with a wine of young age; the second and fifth criaderas were part of a dynamic aging system.

\section{Wine Conditions}

In this study the wine inoculated with the flor yeast strains was obtained from a winery in the Jerez region (Cadiz, Spain). It was a dry (fino) sherry wine, previously fermented and fortified, with the following characteristics: $15.52 \pm 0.18 \%$ (v/v) ethanol, $81.8 \pm 10.3 \mathrm{mg} / \mathrm{L}$ acetaldehyde, $0.38 \pm 0.02 \mathrm{~g} / \mathrm{L}$ volatile acidity and $\mathrm{pH} 3 \pm 0.04$ (avg $\pm \mathrm{SD}$ of the different samples supplied).

\section{Biological Aging of Sherry Wine in Pure Cultures}

Yeast cells were grown in $200 \mathrm{~mL}$ YPD $(1 \%$ yeast extract, $2 \%$ peptone, $2 \%$ glucose) at $30^{\circ} \mathrm{C}$ for $24 \mathrm{~h}$, collected by centrifugation at $4000 \mathrm{rpm}$ for $5 \mathrm{~min}$, washed once with sterile water and resuspended in a known volume of sherry wine. The numbers of total and viable cells were counted under an epifluorescent microscope according to the method previously described [11]. Vessels containing $18 \mathrm{~L}$ of sherry wine, sterilized by filtration, were inoculated with $1 \times 10^{6}$ viable cells $/ \mathrm{mL}$ in order to form the flor velum biofilm. The vessel mouth was plugged with a mosquito net and vessels were kept in the dark at $18^{\circ} \mathrm{C}$. The specific surface (surface/v ratio) in these vessels was $0.052 / \mathrm{cm}$. When the alcohol content dropped below $13.5 \%$, a volume of $6 \mathrm{~L}$ of sherry wine $(1 / 3 \mathrm{vol})$ was removed and then replaced with an equal volume of fresh sherry wine containing distilled alcohol in order to adjust the ethanol concentration in the vessel to $15.5 \%$ and to refresh the aging wine. This sequence of operations imitates the drawing-off and refilling procedures of an industrial dynamic system for sherry wine aging. Seven of the pure cultures were grown for 180 days, while the culture of the VPDN strain was maintained for 310 days.

\section{Analytical Parameters}

Ethanol concentration, acetaldehyde concentration and volatile acidity were analyzed in each culture once a week. Ethanol was quantified according to the transflexion technique using the Infra-analyzer ${ }^{\mathrm{TM}} 450$ system from Techni$\operatorname{con}^{\mathrm{TM}}$ (New York, USA), according to the manufacturer's instructions. Volatile acidity was determined by flow injection (FIA) in an Auto-analyzer ${ }^{\mathrm{TM}}$ II from Technicon ${ }^{\mathrm{TM}}$ (New York, USA), according to the method previously described by Owades and Dono [12], and acetaldehyde concentration was then calculated according to the procedure recommended by the OIV [13].

Table 1. Strains of Saccharomyces Cerevisiae Flor Yeast Used in This Study

\begin{tabular}{|l|l|l|l|}
\hline \multirow{2}{*}{ Strains } & \multicolumn{2}{|c|}{ Molecular patterns $^{*}$} \\
\cline { 2 - 3 } & \multicolumn{1}{|c|}{ Karyotype $^{a}$} & \multicolumn{1}{|c|}{ Origin } \\
\hline \hline B10 & I & A1H1R1 & Sherry wine yeast strain isolated from Añada A [5] \\
\hline BS13 & I & A1H1R1 & Sherry wine yeast strain isolated from Añada A [5] \\
\hline BS24 & I & A3H1R1 & Sherry wine yeast strain isolated from Añada A [5] \\
\hline CHS7 & III & A2H2R2 & Sherry wine yeast strain isolated from Añada B [5] \\
\hline CHS24 & III & A2H2R2 & Sherry wine yeast strain isolated from 2 ${ }^{\text {nd }}$ criadera [4] \\
\hline B17 & III & A2H2R2 & Sherry wine yeast strain isolated from Añada B [5] \\
\hline CHS15 & XXII & A2H3R3 & Sherry wine yeast strain isolated from 5 ${ }^{\text {th }}$ criadera [4] \\
\hline VPDN & XIII & A5H5R6 & $\begin{array}{l}\text { Wine yeast strain isolated from the velum developed by 11763 (obtained from the } \\
\text { CECT) in sherry wine pure culture under laboratory conditions (University of Cadiz) }\end{array}$ \\
\hline
\end{tabular}

${ }^{a}$ Electrophoretic karyotypes were obtained by PFGE. ${ }^{b}$ Different mitochondrial RFLP patterns were obtained by using endonucleases AluI, (patterns A1, A2, A3 and A5); HinfI (patterns H1, H2, H3 and H5) and RsaI (patterns R1, R2, R3 and R6) 


\section{RESULTS AND DISCUSSION}

\section{Analysis of the Biofilm Behaviour of the Flor Yeast Strains}

For the study of enological behaviour, we used the strains listed in Table 1, which had previously been characterized by molecular techniques, to enable a microbiological control procedure to be implemented. The activity of the biofilm growing on the surface of the aging wine during the period of study was monitored by recording two aging indexes: acetaldehyde concentration and volatile acidity (expressed as the concentration of acetic acid). In general, the cultures exhibited a pattern of irregular increase in acetaldehyde concentration (with alternating periods of accumulation and consumption), and a decrease in volatile acidity (Table 2). These variations indicated differences in the behaviour of the biofilm covering the aging wine. In the group of flor yeast strains with chromosomal DNA and mtDNA pattern 'III, A2H2R2', the strains CHS7 and B17 showed similar and lower acetaldehyde concentration than CHS24 in pure culture (Table 2). This difference could be associated with the origin of the strains, as has been shown in other studies [14], because CHS24 was from a dynamic system $\left(2^{\text {nd }}\right.$ criadera, see Table 1) and CHS7 and B17 were isolated from a static system (Añada B) in which the wine was younger. However, a mechanism for adaptive evolution in flor yeast has previously been reported [15]. It is possible that these two strains are less well adapted than CHS24 to biological aging conditions and, for this reason, their behaviour is different and they produce a lower acetaldehyde concentration. The strains B10 and BS13 with molecular pattern 'I, A1H1R1' and the same origin (Añada A) also showed similar behaviour, and the acetaldehyde concentration in the wine was higher than with the strains with patterns 'III, A2H2R2' isolated from Añada B. It should be noted that the acetaldehyde concentration in the wine with BS24 was lower than in those with B10 and BS13 (Table 2), which had the same electrophoretic karyotype but a different $m t$ DNA-RFLP pattern.

Although the strain CHS15 was different in respect of both its molecular pattern and origin, in comparison with the other strains isolated from sherry wines, that strain generated about $300 \mathrm{mg} / \mathrm{L}$ of acetaldehyde. This was similar to the value corresponding to BS13. Finally, the VPDN biofilm produced the most acetaldehyde in the wine. Only minor differences in volatile acidity were found between the different pure cultures (Table 2). Such differences did not produce any noticeable effect on the properties of the aged wine. Among all the yeast biofilms, average values of volatile acidity were less than $0.18 \mathrm{~g} / \mathrm{L}$, which has been described as a very low (acceptable) value in sherry wines [16].

\section{Kinetics of Biofilm Growth and Acetaldehyde Accumula- tion in the Wine}

For the eight pure cultures assayed, the process of development of the flor velum biofilm was similar, with two main stages being observed: phase I or velum formation (PhI) and phase II or velum maintenance (PhII). PhI started when the first groups of yeast cells appeared on the surface of the wine (a few days after the inoculation was performed) and lasted until the whole surface was covered by a thin film of yeast cells. This phase was characterized by exponential cell growth, during which the cell viability remained higher than $90 \%$ in most of the cultures, and its duration, ranging from 9 to 21 days, was found to be strain-dependent (Table 3). Then a rapid transition to PhII took place: the yeast biofilm was still growing but suddenly became thicker. PhII could be considered a stationary phase in which the thick biofilm covering the whole surface of the wine was composed of cells fewer than $10 \%$ of which were viable, and that situation remained unchanged. The periodic operations of drawing-off and refilling fresh wine, carried out in imitation of the industrial procedures, produced breaks in the velum, causing mixing and dilution of the compounds, such as acetaldehyde, in the aging wine. We performed these enological practices repeatedly during the experiments, and each time it led to a short-term transition to a renewed phase I (secondary phase I, $\left.2^{\text {nd }} \mathrm{PhI}\right)$, which was followed by a renewed phase II $\left(2^{\text {nd }}\right.$ PhII). This general pattern of evolution of acetaldehyde concentration can be observed for the case of strain BS24 in Fig. (1).

Table 2. Acetaldehyde Concentration and Volatile Acidity in Eight Different Pure Cultures During Biological Aging. Average, Standard Deviation, Maximum and Minimum Values for the Period of Study are Shown

\begin{tabular}{|c|c|c|c|c|c|c|c|}
\hline \multirow{2}{*}{ Culture } & \multicolumn{3}{|c|}{ Acetaldehyde (mg/L) } & \multicolumn{3}{|c|}{ Volatile Acidity (g/L) } & \multirow{2}{*}{$\begin{array}{c}\text { Total } \\
\text { Measures }\end{array}$} \\
\hline & Av. \pm SD & Max & Min & Av. \pm SD & Max & Min & \\
\hline B17 & $113 \pm 11$ & 130 & 100 & $0.08 \pm 0.03$ & 0.17 & 0.05 & 27 \\
\hline CHS7 & $140 \pm 27$ & 187 & 99 & $0.12 \pm 0.06$ & 0.23 & 0.06 & 25 \\
\hline BS24 & $176 \pm 45$ & 238 & 95 & $0.10 \pm 0.04$ & 0.19 & 0.06 & 24 \\
\hline CHS24 & $203 \pm 35$ & 246 & 162 & $0.10 \pm 0.04$ & 0.17 & 0.05 & 25 \\
\hline B10 & $233 \pm 72$ & 470 & 80 & $0.17 \pm 0.06$ & 0.35 & 0.06 & 24 \\
\hline BS13 & $281 \pm 65$ & 387 & 217 & $0.15 \pm 0.08$ & 0.30 & 0.07 & 25 \\
\hline CHS15 & $306 \pm 70$ & 472 & 234 & $0.10 \pm 0.03$ & 0.16 & 0.06 & 26 \\
\hline VPDN & $479 \pm 147$ & 740 & 157 & $0.16 \pm 0.05$ & 0.30 & 0.08 & 37 \\
\hline
\end{tabular}

${ }^{\mathrm{a}}$ Measures were made each seven days throughout the process 
Table 3. Average Rates of Acetaldehyde Accumulation During Different Phases of the Biofilm Growth, Described in the Text. The Average Duration (in Days) of These Phases in Each Culture is Also Presented

\begin{tabular}{|c|c|c|c|c|}
\hline \multirow[b]{2}{*}{ Culture } & \multicolumn{2}{|c|}{ Av. Rate of Acetaldehyde Accumulation (mg/L per day) } & \multicolumn{2}{|c|}{ Av. Duration of Phase (days) } \\
\hline & Phase $\mathrm{I}^{\mathrm{a}}$ & Phase II & Phase $^{\mathrm{b}}$ & Phase II \\
\hline B10 & $17.5(30.0)$ & -17.0 & $8.8(13)$ & 13.0 \\
\hline BS13 & $13.1(25.9)$ & -9.6 & $15.2(12)$ & 14.0 \\
\hline BS24 & $13.4(18.3)$ & -5.1 & $8.7(7)$ & 15.4 \\
\hline CHS15 & $22.2(36.0)$ & -13.6 & $15.0(11)$ & 17.5 \\
\hline CHS7 & $11.7(22.2)$ & -5.0 & $10.8(5)$ & 17.5 \\
\hline CHS24 & $14.3(33.0)$ & -6.0 & $25.0(5)$ & 9.3 \\
\hline B17 & 5.9 (13.5) & -3.2 & $13.4(4)$ & 9.0 \\
\hline VPDN & $8.9(9.4)$ & c & 20.8 (53) & c \\
\hline
\end{tabular}

${ }^{a}$ Specific average rates were computed including successive PhI's. The accumulation of the acetaldehyde in each PhI was calculated measuring the increase from each operation of drawing-off and refilling until the value drops, and taking into account the days after. Values in brackets correspond to the acetaldehyde accumulation during the initial phase of velum formation (PhI). ${ }^{b}$ The duration of the initial $\mathrm{PhI}$ and each successive PhI was determined based on both acetaldehyde concentrations and observation of the biofilms. The higher values in brackets correspond to the initial phase of velum formation ( $\left.1^{\text {st }} \mathrm{PhI}\right)$, whereas the average duration of phase was computed including the initial and successive PhI's. In the case of VPDN, the periods of acetaldehyde accumulation were only interrupted by the operations of drawing-off and refilling with new wine. ${ }^{\text {T}}$ The biofilm of the VPDN strain remained in the phase of velum formation (PhI) during the whole period of study.

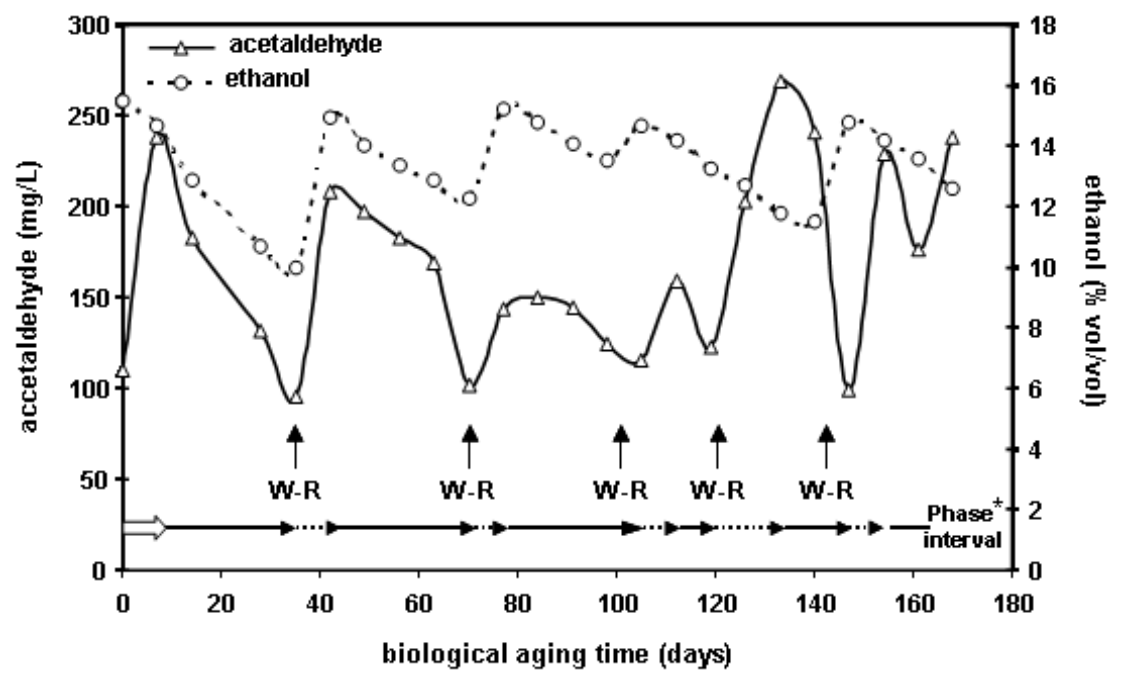

Fig. (1). Evolution of ethanol and acetaldehyde concentrations in the BS24 culture. Times of the operations of drawing-off and refilling (W-R) with fresh fortified wine are indicated by vertical arrows. ${ }^{*}$ Duration of the phases is represented by different horizontal arrows: $\Rightarrow$, PhI; $\cdots \cdots>, 2^{\text {nd }}$ PhI induced by operations of drawing-off and refilling; $\rightarrow$, PhII or $2^{\text {nd }} \mathrm{PhII}$. Figure shows that $\mathrm{Ph}$ I and $2^{\text {nd }} \mathrm{PhI}$ corresponded to periods of acetaldehyde accumulation, whereas $\mathrm{PhII}$ and $2^{\text {nd }} \mathrm{PhII}$ corresponded to acetaldehyde depletion.

However, each culture displayed a relatively high rate of acetaldehyde accumulation during the initial PhI (Table 3), compared with the rate occurring during successive $2^{\text {nd }}$ PhI's, which was lower.

In contrast, during PhII, acetaldehyde no longer accumulated in the wine but was depleted at a strain-dependent rate (Table 3). The decrease in acetaldehyde concentration observed in PhII is in agreement with previous results, indicating that once the thicker velum is formed, cell biosynthesis stops [17]. Hence the concentration of acetaldehyde decreases until a refilling with new fortified wine leads to a temporary transition to a $2^{\text {nd }} \mathrm{PhI}$.
It should also be noted that the VPDN strain behaved differently from the other strains during aging. We observed that it formed a thin biofilm which did not completely cover the surface of the wine, and this partial biofilm remained in PhI. Moreover, the biofilm in the $1^{\text {st }} \mathrm{PhI}$ I took longer to form than in the other strains - about 53 days (Table 3). This culture showed an almost constant rate of acetaldehyde accumulation (around $9 \mathrm{mg} / \mathrm{L}$ per day, Table 3), although a high average concentration of $479 \mathrm{mg} / \mathrm{L}$ was reached (Table 2). After the first period of velum formation, the proportion of viable cells decreased slowly to values of about $40 \%$ of 
the total, whereas the cell viability of other strains showed a faster and steeper reduction (to values of less than 10\%).

The CHS15 culture also showed acetaldehyde accumulation at a relatively high rate (36 $\mathrm{mg} / \mathrm{L}$ per day) during the first 11 days. The transition to PhII then occurred, and the acetaldehyde concentration decreased (to an average rate of $13.6 \mathrm{mg} / \mathrm{L}$ per day) until the next operation of drawing-off and refilling with fresh wine induced a $2^{\text {nd }} \mathrm{PhI}$. The average duration of the repeated $2^{\text {nd }}$ PhI's was 15 days, during which acetaldehyde accumulation increased again to an average rate of $22.2 \mathrm{mg} / \mathrm{L}$ per day (Table 3 ). The high level of acetaldehyde accumulation recorded in wine aged by CHS15 (Table 2) is possibly because CHS15 was observed to be able to release a relatively large amount of acetaldehyde into the wine during PhI. Nevertheless, the acetaldehyde accumulation in the CHS15 culture was not as high as that in the VPDN culture, because the CHS15 culture remained in PhII for a long period, during which time the cell viability was extremely low (below 10\%) and acetaldehyde was being depleted.

The cell viability of the VPDN culture during the sherry wine aging process was higher and more constant compared with the other strains of this study (data not shown), which may be associated with the strain's general capacity for producing higher acetaldehyde concentrations, as observed by other researchers $[18,19]$. Therefore, the high level of acetaldehyde in the VPDN culture could be due to its capacity to resist the high acetaldehyde concentration in the assay conditions. Its cells remained for a longer time in PhI, in a slow but exponential growth phase, releasing acetaldehyde at a low but constant rate, and that explains the high acetaldehyde concentration recorded in the wine aged by this strain (Table 2).

In the cultures of the CHS15, B10 and BS13 strains, the time needed for the biofilm to cover the surface of the wine after the inoculation coincided with the duration of PhI. Strains B10 and BS13 behaved similarly in the development of the biofilm, also accumulating high concentrations of acetaldehyde (Table 3). However, the formation of the biofilm took about 14 days in BS24, CHS7, CHS24 and B17 whereas acetaldehyde accumulation stopped several days before this was completed (Table 3 ). It has been reported that acetaldehyde exerts deleterious effects on yeast cells when its concentration reaches a threshold, which is variable according to the strain $[19,20]$. Presumably, action to mix the culture and so homogenize the acetaldehyde concentration in the whole culture volume would be advantageous. This hypothesis is supported by the fact that, in these cultures, the duration of the $2^{\text {nd }} \mathrm{PhI}$ induced by a refilling operation, which produced some mixing, was longer than that of the $1^{\text {st }}$ PhI (Table 3).

\section{Design of Strategies to Improve Wine Aging}

It seems reasonable that the differences in enological performance, particularly in growth kinetics and acetaldehyde productivity, shown in the present results can be associated with the different genetically-characterized strains of the $S$. cerevisiae flor yeast. These strains were isolated from fino-type sherry wines, in which acetaldehyde is considered to be the most influential component in the organoleptic properties of the finished wine [16]. Thus, when a sherry winery is seeking to improve the biological aging process, it is necessary to select those yeast strains that generate larger amounts of acetaldehyde and that form the initial biofilm more rapidly after inoculation, partly to accelerate the aging biological process and partly to enhance the organoleptic properties. With these objectives, the VPDN strain is likely to be a candidate in a selection program for a particular industrial environment and, in turn, the newly-selected VPDN strain could be used as inoculum. This strain, which shows unique chromosomal and $m t$ DNA patterns, produces the continuous accumulation of more-than-sufficient amounts of acetaldehyde and therefore its inoculation in the blending stages would accelerate the biological aging of the wine. Nevertheless, the biofilm activity of the VPDN pure culture is too low to cover the whole wine surface, in an industrial environment; this inadequacy could lead to excessive oxidation of the wine. Moreover, its rate of acetaldehyde accumulation during $\mathrm{PhI}$ was very low. Taking all these factors into consideration, the VPDN strain should be used in combination with other yeast strains, to compensate for those disadvantages of the VPDN strain. The utilisation of wine yeast in mixed cultures is a practice that has been proposed for inoculating alcoholic fermentations [21]. The genetic manipulation of flor yeast to improve sherry wine production has also been proposed [14], but the current regulations in the Jerez region do not permit the use of genetically modified yeasts.

The inoculation of the mixed cultures is therefore suggested. For example, the CHS15, B10 or BS13 strains could be used initially, not only to accumulate acetaldehyde at a high rate during $\mathrm{PhI}$ but also to establish a complete biofilm across the whole wine surface. Prior to the transition to PhII, the wine could be inoculated with the VPDN strain, to continue the accumulation of acetaldehyde so that the wine is fully aged more rapidly, with optimum properties. Thus, further studies on the behaviour of different flor yeast strains in mixed cultures are considered of interest and of industrial relevance.

\section{CONCLUSIONS}

In this study, we have found that flor yeast strains of $S$. cerevisiae with identical chromosomal and mitochondrial DNA patterns, and the same origin, showed a more closelyrelated enological behaviour, but no correspondence was found between a particular molecular pattern and physiological behaviour in, for example, the concentration of acetaldehyde produced in the wine. The kinetics of biofilm growth was similar for the strains assayed. We observed that each pure culture displayed specifically a high rate of acetaldehyde accumulation during $\mathrm{PhI}$ and a decrease in this parameter in PhII. No correspondence was observed between rates of acetaldehyde accumulation and consumption, and the molecular pattern of the strain. However, taking into account other parameters including the time (in days) taken for the initial biofilm to form, the strains with identical pattern, such as B10 and BS13 (pattern 'I, A1H1R1') and CH7, CHS24 and B17 (pattern 'III, A2H2R2') do show similar behaviour. The approach described in this article is important for the entire Enology sector, because there is little information available on the design of strategies for using selected flor yeast strains to improve wine aging on the industrial scale. 
Those strains that generate a high concentration of acetaldehyde and form a complete biofilm more rapidly will be good candidates for further assays to be carried out in industrial conditions.

\section{CONFLICT OF INTEREST}

The authors confirm that this article content has no conflicts of interest.

\section{ACKNOWLEDGEMENTS}

This work was supported by PAI grant to the group BIO219.

\section{REFERENCES}

[1] Martínez P, Codón AC, Pérez L, Benítez T. Physiological and molecular characterization of flor yeast: polymorphism of flor yeast populations. Yeast 1995; 11:1399-411.

[2] Esteve-Zarzoso B, Peris-Torán MJ, García-Maiquez E, Uruburu F, Querol A. Yeast population dynamics during the fermentation and biological aging of sherry wines. Appl Environ Microbiol 2001; 67: 2056-61.

[3] Ibeas JI, Lozano I, Perdigones F, Jiménez J. Dynamics of flor yeast populations during the biological aging of sherry wines. Am J Enol Vitic 1997; 48: 75-9.

[4] Mesa JJ, Infante JJ, Rebordinos L, Cantoral JM. Characterization of yeasts involved in the biological ageing of sherry wines. Food Sci Technol 1999; 32: 114-20.

[5] Mesa JJ, Infante JJ, Rebordinos L, Sánchez JA, Cantoral JM. Influence of the yeast genotypes on enological characteristics of sherry wines. Am J Enol Vitic 2000; 5:15-21.

[6] Rodríguez ME, Infante JJ, Molina M, et al. Genomic characterization and selection of wine yeast to conduct industrial fermentations of a white wine produced in a SW Spain winery. J Appl Microbiol 2010; 108: 1292-302.

[7] Moreno JA, Zea L, Moyano L, Medina M. Aroma compounds as markers of the changes in Sherry wines subjected to biological ageing. Food Control 2005; 14: 333-8.
[8] Cortés MB, Moreno JJ, Zea L, Moyano L, Medina M. Response of the aroma fraction in sherry wines subjected to accelerated biological aging. J Agric Food Chem 1999; 47: 3297-302.

[9] Budroni M, Giordano G, Pinna G, Farris GA. A genetic study of natural flor strains of Saccharomyces cerevisiae isolated during biological ageing from Sardinian wines. J Appl Microbiol 2000; 89: 657-62.

[10] Charpentier C, Colin A, Alais A, Legras JL. French Jura flor yeast: genotype and technological diversity. Antonie van Leeuwenhoek 2009; 95: 263-73.

[11] King LM, Schisler DO, Ruocco JJ. Epifluorescent method for detection of nonviable yeast. J Am Soc Brew Chem 1981; 39(2): 254.

[12] Owades JL, Dono JM. Determination of volatile acids in wine by micro-diffusion. Am J Enol Vitic 1968 ; 19: 46-51.

[13] OIV (Office International de la Vigne et du Vin). Recueil des méthodes internationals d'analyse des vins et de moûts. Paris, 1979 France.1979.

[14] Aranda A, Querol A, del Olmo M. Correlation between acetaldehyde and ethanol resistence and expresion of HSP genes in yeast strains isolated during the biological aging of sherry wines. Arch Microbiol 2002; 177: 304-12.

[15] Infante JJ, Dombek KM, Rebordinos L, Cantoral JM, Young ET. Genome-wide amplifications caused by chromosomal rearrangements play a major role in the adaptive evolution of natural yeast. Genetics 2003; 165: 1745-59.

[16] Martínez P, Valcárcel MJ, Pérez L, Benítez T. Metabolism of Saccharomyces cerevisiae flor yeasts during fermentation and biological aging of fino sherry: by-products and aroma compounds. Am J Enol Vitic 1998; 49: 240-50.

[17] Berlanga TM, Atanasio C, Mauricio JC, Ortega JM. Influence of aeration on the physiological activity of flor yeasts. J Agric Food Chem 2001; 49: 3378-84.

[18] Liu S, Pilone GJ. An overview of formation and roles of acetaldehyde in winemaking with emphasis on microbiological implications. Int J Food Sci Technol 2000; 35: 49-61.

[19] Martínez P, Pérez-Rodríguez L, Benítez T. Evolution of flor yeast population during the biological aging of fino sherry wine. Am J Enol Vitic 1997; 48: 160-8.

[20] Ristow H, Seyfarth A, Lochmann ER. Chromosomal damage by ethanol and acetaldehyde in Saccharomyces cerevisiae as studied by pulsed field gel electrophoresis. Mutat Res 1995; 326:165-70.

[21] Fleet GH. Wine yeasts for the future. FEMS Yeast Res 2008; 8: 979-95.

(c) Rodríguez et al.; Licensee Bentham Open.

This is an open access article licensed under the terms of the Creative Commons Attribution Non-Commercial License (http://creativecommons.org/licenses/ by-nc/3.0/) which permits unrestricted, non-commercial use, distribution and reproduction in any medium, provided the work is properly cited. 